\title{
Biallelic SORD pathogenic variants cause Chinese patients with distal hereditary motor neuropathy
}

\author{
Hai-Lin Dong $\mathbb{1}^{1}$, Jia-Qi Li ${ }^{1}$, Gong-Lu Liu ${ }^{1,2}$, Hao Yu ${ }^{1}$ and Zhi-Ying Wu $\mathbb{( D D}^{1 凶}$
}

Sorbitol dehydrogenase gene (SORD) has been identified as a novel causative gene of recessive forms of hereditary neuropathy, including Charcot-Marie-Tooth disease type 2 and distal hereditary motor neuropathy (dHMN). Our findings reveal two novel variants (c.404 A > G and c.908 + 1 G >C) and one known variant (c.757delG) within SORD in four Chinese dHMN families. Ex vivo CDNA polymerase chain reaction confirmed that c.908 $+1 \mathrm{G}>\mathrm{C}$ variant was associated with impaired splicing of the SORD transcript. In vitro cell functional studies showed that c.404 A $>\mathrm{G}$ variant resulted in aggregate formation of SORD and low protein solubility, confirming the pathogenicity of SORD variants. We have provided more evidence to establish SORD as a causative gene for dHMN.

npj Genomic Medicine (2021)6:1 ; https://doi.org/10.1038/s41525-020-00165-6

The distal hereditary motor neuropathy (dHMN) is a group of clinically and genetically heterogeneous inherited peripheral neuropathy, characterized by slowly progressive distal limb muscle weakness and atrophy, tendon areflexia, and foot deformities ${ }^{1}$. The dHMN often shares a phenotypic overlap with the axonal form of Charcot-Marie-Tooth disease type 2 (CMT2) with no or minor sensory neuropathy. In addition, it has an overlap with CMT2 in causative genes, such as GARS, IGHMBP2, AARS, and HARS genes ${ }^{2,3}$. Although at least 30 disease-causing genes for $\mathrm{dHMN}$ have been identified (http://neuromuscular.wustl.edu), the genetic diagnosis remains elusive for $70-80 \%$ of affected individuals ${ }^{4,5}$.

Sorbitol dehydrogenase gene (SORD) encodes a 357 amino acid protein and the protein acts as a key enzyme in the polyol pathway. Very recently, variants in SORD have been identified as a novel cause, and also the most common form in individuals affected by CMT2 or $\mathrm{dHMN}^{6}$. Totally, 45 affected individuals from 38 families, including 23 CMT2 (3 from China), $18 \mathrm{dHMN}$, and 4 intermediate CMT patients were identified to carry homozygous or heterozygous c.757delG (p.Ala253GlnfsTer27) variant in SORD.

Here, we report the identification of two novel variants (c.404 A $>\mathrm{G}$ and c.908 + 1 G >C) and one known variant (c.757delG) in $S O R D$ in four Chinese dHMN families. This report further establishes SORD as a disease-causing gene for dHMN, and expands both the phenotypic and mutational spectra of the SORD-associated hereditary neuropathy.

To investigate the underlying causative gene in Chinese CMT2 and dHMN, we have collected a cohort of $20 \mathrm{CMT} 2$ and $9 \mathrm{dHMN}$ patients without known genetic cause. Whole-exome sequencing (WES) was conducted on all the patients. After analyzing and filtering, we found three dHMN patients from family 1, 2, and 3 carrying the known homozygous c.757delG (p.Ala253GInfsTer27) variant in SORD. Furthermore, two novel variants were detected in the proband (dHMN) of family 4 , including a missense variant c.404 A > G (p.His135Arg) and a splicing variant c.908+1 G > C. The results of Sanger sequencing confirmed the presence of the identified variants in corresponding individuals and showed SORD variants co-segregating with the disease in four families, respectively (Fig. 1a, b). They were absent in several general population frequency databases, such as the 1000 Genomes Project, Exome Aggregation Consortium (ExAC), and the Genome Aggregation Database (gnomAD). To ascertain the pathogenicity of these two novel variants, we analyzed them using several bioinformatic algorithms. The p.His135Arg was predicted to be damaging by SIFT (score 0.001), disease-causing by MutationTaster (score 1.0), probably damaging by PolyPhen-2 (score 1.0), and damaging by CADD (score 24.7). The c.908 $+1 \mathrm{G}>\mathrm{C}$ variant was located within the highly conserved donor splice site of exon 8 and predicted to cause abnormal splicing by abolishing the donor splice site of exon 8 (dbscSNV ada-score $=0.9999$, rf-score $=$ 0.934).

To confirm whether the c.908 $+1 \mathrm{G}>\mathrm{C}$ variant affects splicing, total RNA was isolated from patient's peripheral blood, and reverse transcribed into cDNA and amplified. Agarose gel electrophoresis showed a normal-sized band and a shorter band in the proband (II-2) of family 4. TA clone and Sanger sequencing of CDNA polymerase chain reaction (PCR) products revealed two abnormal spliced SORD transcripts, an amplicon with four nucleotides inserted prior to exon 9 due to the utilization of a cryptic intronic donor splice site (p.Trp304*), and an amplicon skipping of 35 base pairs at the $3^{\prime}$ end of exon 8 that caused premature stop codon p.Asp293AlafsTer21 (Fig. 1c).

To explore the influence of the variant c.757delG (p.Ala253GlnfsTer27) on the function of SORD gene, we used quantitative PCR to examine SORD mRNA levels in peripheral blood from the proband of family 2 (II-1), his father (I-2), and the healthy control. Compared to the healthy control, the patient carrying a homozygous c.757delG (p.Ala253GInfsTer27) variant showed a significant reduction in SORD mRNA expression levels of $\sim 70-80 \%$ when normalized to the control gene GAPDH $(p<0.001)$. His father carrying a heterozygous c.757delG (p.Ala253GlnfsTer27) variant exhibited a milder decrease of $\sim 45-50 \%$ in mRNA levels $(p<0.01$; Fig. 1d). It suggested that mRNAs of patients with the c.757delG (p.Ala253GInfsTer27) variant were unstable on account of the nonsense-mediated mRNA decay (NMD).

\footnotetext{
${ }^{1}$ Department of Neurology and Research Center of Neurology in Second Affiliated Hospital, and Key Laboratory of Medical Neurobiology of Zhejiang Province, Zhejiang University School of Medicine, Hangzhou, China. ${ }^{2}$ Department of Neurology and Institute of Neurology, Huashan Hospital, Shanghai Medical College, Fudan University, Shanghai, China. ${ }^{凶}$ email: zhiyingwu@zju.edu.cn
} 
a

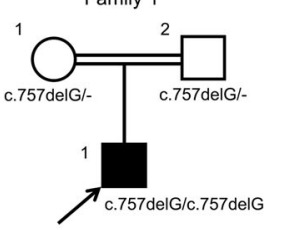

b

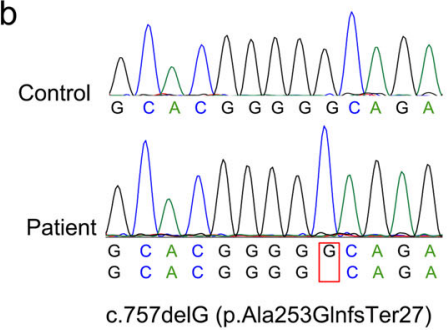

C

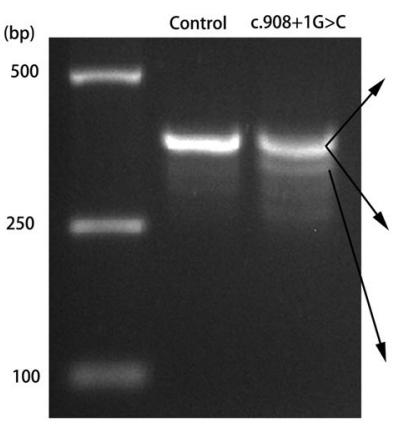

e

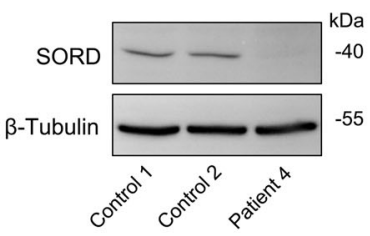

f

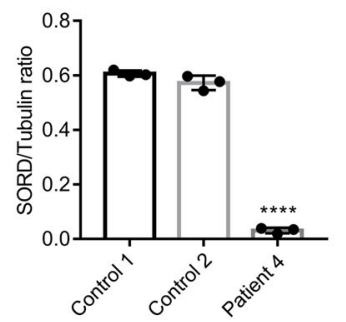

$\mathrm{h}$
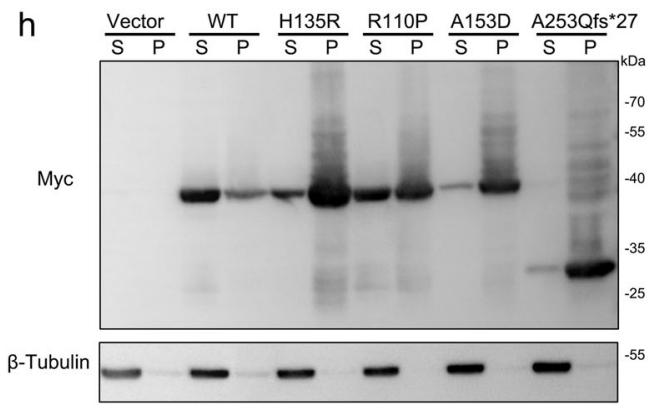

We used fibroblast of patient 4 with compound c.908 + $1 \mathrm{G}>$ C/p.His135Arg variants as a model to assess the effects of two variants on SORD protein expression. Western blot showed that SORD protein expression was significantly decreased compared to two healthy controls (Fig. 1 e, f). A previous study has
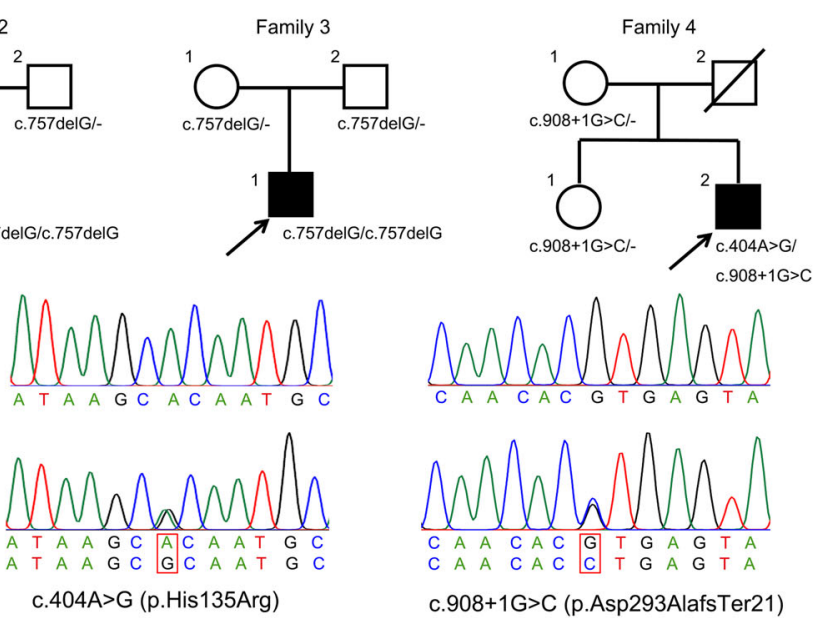

c.908+1G>C (p.Asp293AlafsTer21)

d

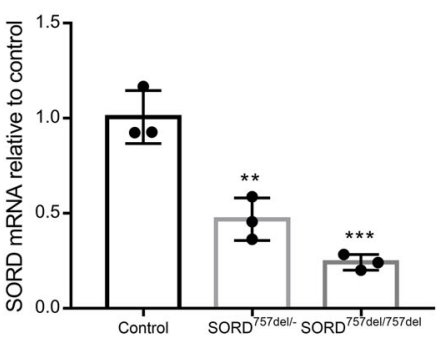

p.Asp293AlafsTer21
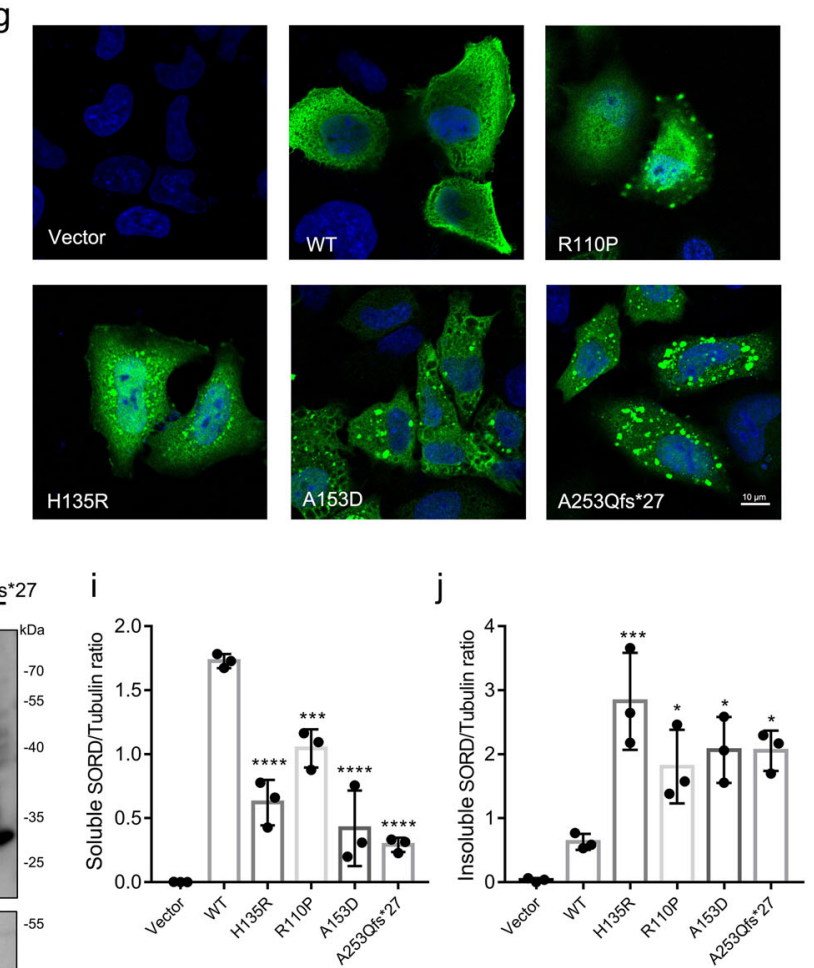

demonstrated that residues $90-110$ and 120-140 in SORD subunit are important for the stability and binding of tetramer ${ }^{7}$. To define whether the variant p.His135Arg and two previously reported missense variants (p.Arg110Pro and p.Ala153Asp) flanking it altered the tetramer-forming interactions, five plasmids expressing 
Fig. 1 Genetic and functional findings in dHMN patients with SORD variants. a Pedigrees of four Chinese dHMN families carrying SORD variants. Circle: females; square: males; open symbol: unaffected; filled symbol: affected; arrow: proband of each family. b Sequencing chromatograms of three variants within SORD. The lower chromatogram represents the variant and the upper one represents the normal sequence. c SORD CDNA products were separated by agarose gel electrophoresis and confirmed by Sanger sequencing. Lane 1: marker; lane 2: control; lane 3: subject II-2 (family 4). d Relative SORD mRNA expression levels from peripheral blood in subjects with a heterozygous or homozygous c.757delG (p.Ala253GInfsTer27) compared with the healthy controls $(n=3$ experimental repeats, data are shown as mean \pm SD. $\left.{ }^{* *} p<0.01 ;{ }^{* * *} p<0.001\right)$. e Fibroblast SORD protein expression levels in two normal controls and patient 4 with compound c.908 $+1 \mathrm{G}>\mathrm{C} / \mathrm{p}$. His135Arg variants. $\mathbf{f}$ The graph shows $\beta$-tubulin normalized SORD expression levels $(n=3$ biological repeats, data are shown as mean \pm SD. **** $p<0.0001)$. g Immunofluorescence analysis of HeLa cells expressing WT or mutant SORD. Cells were stained by mouse anti-Myc antibody followed by anti-mouse Alexa Fluor 488 (green)-conjugated secondary antibody and DAPI (blue). Scale bars $=10 \mu \mathrm{m}$. $\mathbf{h}$ Soluble (S) and insoluble (P) protein fractions per variant with western blot probing for Myc-SORD and $\beta$-Tubulin (loading control). The graphs show $\beta$-tubulin normalized Myc-SORD expression levels per variant in soluble protein fractions (i) and insoluble protein fractions $(j)(n=3$ biological repeats, data are shown as mean $\left.\pm \mathrm{SD} .{ }^{*} p<0.05 ;{ }^{* * *} p<0.001 ;{ }^{* * * *} p<0.0001\right)$. All blots were derived from the same experiment and were processed in parallel.

wild type (WT), R110P, H135R, A153D, or A253Qfs*27 protein were constructed. Immunofluorescence experiment in HeLa cells transiently expressing WT or mutant SORD showed that WT SORD displayed diffuse intracellular staining, whereas mutant SORD displayed granules throughout the cytosol (Fig. 1g). To confirm the presence of SORD aggregates, we investigated the soluble and insoluble SORD fractions by western blot. The levels of mutant SORD protein were markedly reduced in the soluble fractions of transfected cells with an increase in the insoluble fractions (Fig. 1h-j). Altogether, these studies provided evidence that p.His135Arg caused aggregate forming of SORD due to probable conformational changes in tetrameric state, which indicated the variant was pathogenic.

Altogether, we identified 4 unrelated dHMN patients harboring biallelic SORD pathogenic variants. The detailed clinical features and nerve conduction studies of these patients were shown in Table 1. All four cases were sporadic and only one (Family 1: II-1) was born of a consanguineous marriage. The average age of disease onset was 12.5 \pm 3.5 years (range 9-16). All affected individuals complained about muscle weakness in their lower limbs during their teenage years, thus having difficulty in walking, running, and squatting down. The disease presented slowly progressive in all the probands. The distal muscle atrophy began in their feet and legs, followed by intrinsic muscles of the hands. As a consequence, they were not able to make fine and complicated movements using their hands. On electrophysiological examination, the nerve conduction studies showed lengthdependent axonal motor neuropathy with marked decrease in the amplitudes of compound muscle action potentials and mild reduce in the motor nerve conduction velocities in lower limb nerves. The sensory nerve conduction velocities and sensory nerve action potentials were in the normal range.

The SORD catalyzes the interconversion between glucose and fructose via sorbitol together with aldose reductase. The polyol pathway is an important alternate route for sugar metabolism. Therefore, it is believed that SORD is involved in the development of diabetic neuropathy ${ }^{8,9}$. To date, eight pathogenic variants in SORD have been identified in CMT2 or dHMN. The variant c.757delG (p.Ala253GlnfsTer27) is the most common cause with a frequency of up to $\sim 10 \%$ in undiagnosed CMT2 or dHMN cases ${ }^{6}$. Similarly, the most common pathogenic variant (c.757delG, p. Ala253GInfsTer27) was identified in three dHMN families in our study, which occupied $33.3 \%$ (3/9) of genetically undefined dHMN cases. In addition, the patient carrying a homozygous c.757delG (p.Ala253GInfsTer27) variant showed a dramatic reduction in SORD mRNA levels. This result was consistent with the finding of the protein expression level in the patient with the c.757delG ( $p$. Ala253GInfsTer27) variant ${ }^{6}$, indicating a NMD mechanism and loss of function of SORD gene. The absence of SORD protein and an increased level of intracellular sorbitol were found in patientderived fibroblasts ${ }^{6}$. Furthermore, synaptic degeneration and progressive motor impairment appeared in Drosophila with loss of SORD orthologs ${ }^{6}$.
In conclusion, we identified SORD pathogenic variants in four dHMN families, further establishing SORD as a disease-causing gene for dHMN. Our findings provide different mutation types of $S O R D$, expanding the spectrum of pathogenic variants in SORD and highlighting the importance of screening SORD variants among undefined hereditary neuropathy patients.

\section{METHODS}

Subjects

A cohort of $20 \mathrm{CMT} 2,9 \mathrm{dHMN}$ patients, and available unaffected relatives were enrolled consecutively in this study between June 4, 2008 and October 6, 2019 from southeastern China. They have been excluded other inherited peripheral neuropathies by genetic screening of the known causative genes in our previous reports ${ }^{10-13}$. Each patient received clinical evaluation and diagnosis from at least two senior neurologists, according to clinical features and electrophysiological studies described in our previous reports ${ }^{11,14}$. In addition, the criteria for the enrollment were as follows: (1) autosomal recessive or sporadic; (2) slowly progressive weakness and atrophy of the distal limb muscles; and (3) electrophysiological results showed preserved or only mildly slowed nerve conduction velocities ( $>38 \mathrm{~m} / \mathrm{s}$ ) and showed primarily axonal damage (CMT2) or a pure motor axonal neuropathy without sensory involvement (dHMN). Written informed consents were obtained from all participants. The study was approved by the ethics board of Second Affiliated Hospital, Zhejiang University School of Medicine, and Huashan Hospital of Fudan University.

Whole-exome sequencing and bioinformatic analyses

Genomic DNA of the probands and unaffected family members were extracted from peripheral blood using a standard kit (Qiagen, Germany). Then the Agilent SureSelect ${ }^{\mathrm{TM}}$ Human All Exome V6 kit (Agilent Technologies Inc, Canada) on an Illumina Hiseq X Ten Analyzer (Illumina, USA) was used to perform WES in all the subjects. The frequency of all the variants in the general population was filtered by the 1000 Genomes Project (https://www.ncbi.nlm.nih.gov/variation/tools/1000 genomes/) ${ }^{15}$, ExAC (http://exac.broadinstitute.org/) ${ }^{16}$, and gnomAD (http://gnomad-old. broadinstitute.org $/)^{17}$. The pathogenicity of the identified variants was analyzed using SIFT (http://sift.jcvi.org/) ${ }^{18}$, PolyPhen-2 (http://genetics.bwh. harvard.edu/pph2//) ${ }^{19}$, MutationTaster (http://www.Mutation taster. org//20, and $\mathrm{CADD}^{21}$. To predict the splicing effects of $c .908+1 \mathrm{G}>\mathrm{C}$ variant, two prediction scores (ada-score and rf-score) were extracted from dbscSNV database ${ }^{22}$. The variant is interpreted as splice-altering if the score is $>0.6$.

\section{Sanger sequencing of SORD and SORD2P}

Exonic and intronic areas of SORD and exon 7 of SORD2P were amplified by PCR followed using the primers described previously ${ }^{6}$. Sanger sequencing was performed to validate the candidate variants analyzed by WES and cosegregation of the pedigrees among all available family members.

RNA isolated, splicing analysis, and quantitative PCR

Total RNA was isolated from peripheral blood treated with RNAiso Plus (Takara, Japan). RNA was reverse transcribed into CDNA using the PrimeScript $^{\mathrm{TM}}$ II 1st Strand CDNA Synthesis Kit (Takara, Japan). For the analysis of the splicing variant, PCR products of CDNA were visualized in 
H.-L. Dong et al.

Table 1. Clinical features of patients carrying SORD variants.

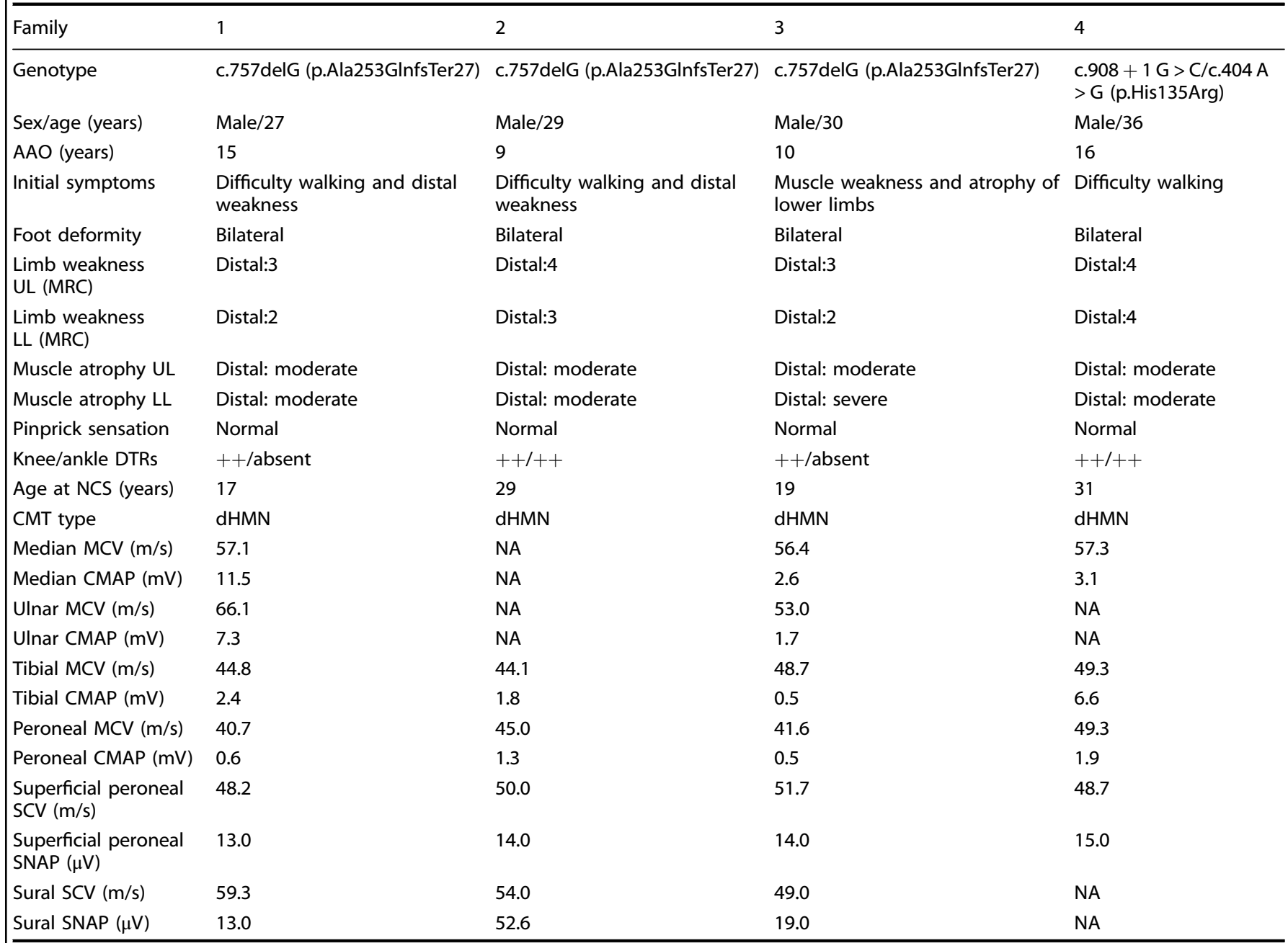

$A A O$ age at onset, UL upper limbs, $L L$ lower limbs, MRC Medical Research Council scale, DTR deep tendon reflex, NCS nerve conduction studies, CMAP compound muscle action potential, MCV motor nerve conduction velocity, SCV sensory nerve conduction velocity, SNAP sensory nerve action potential, NA not available.

agarose gels to study the transcribed pattern and then cloned into pGMsimple-T TA vector (TIANGEN, China). The vector was transformed into DH5a cells (TIANGEN, China), and individual colonies were examined and sequenced. The targeted and control region were amplified respectively using the following primers: SORD forward: $5^{\prime}$-CAAGCCCAACAACC TTCCC3', SORD reverse: 5'-TGAGGGCAGAGCCCATTAAC-3', GAPDH forward: 5'GGAGCGAGATCCCTCCAAAAT-3'; and GAPDH reverse: 5'-GGCTGTTGTCA TACT TCTCATGG-3'. The quantitative PCR was run and analyzed on an ABI StepOne real-time PCR system (Thermo Fisher, USA).

Expression plasmids

The WT full-length coding region of human SORD CDNA (NM_003104.6) was cloned into PCMV-Myc-N terminal tag vector (Clontech, USA). Three missense SORD variants (p.Arg110Pro, p.His135Arg, and p.Ala153Asp) and the nonsense p.Ala253GInfsTer27 variant reported previously were introduced into WT SORD construct, using PCR mutagenesis (TOYOBO, China).

\section{Cell culture and plasmids transfection}

Primary fibroblasts were obtained from skin biopsy of patient 4 and two normal controls and grown in Dulbecco's Modified Eagle Medium (Gibco, USA) supplemented with $10 \%$ fetal bovine serum (Gibco, USA) at $37^{\circ} \mathrm{C}$ in $5 \% \mathrm{CO}_{2}$. HEK-293T and Hela cell are from CBTCCCAS (The Cell Bank of Type
Culture Collection of Chinese Academy of Sciences). For immunofluorescence analysis, Hela cells were cultured in glass-bottomed 24-well plates and transfected with $500 \mathrm{ng}$ of plasmid DNA, using Lipofectamine 3000 reagent (Invitrogen, USA) for $24 \mathrm{~h}$. For protein expression analysis, HEK$293 T$ cells were cultured in $6 \mathrm{~cm}$ dishes and transfected with $4000 \mathrm{ng}$ of plasmid DNA, using Lipofectamine 3000 reagent for $48 \mathrm{~h}$.

Immunofluorescence analysis

Hela cells transiently transfected with Myc-tagged SORD were cultured in glass-bottomed dishes. Cells were fixed with $4 \%$ paraformaldehyde for 20 min, permeabilized, and blocked in PBS containing 0.1\% TritonX-100 (Beyotime Biotechnology, China) and 5\% bovine serum albumin (Sigma, USA) for $60 \mathrm{~min}$. Cells were then incubated with anti-Myc (1:1000, SAB2702192, Sigma, USA) antibody at $4{ }^{\circ} \mathrm{C}$ overnight, followed by secondary anti-mouse IgG Alexa Fluor 488 antibodies (1:1000, Life Technologies, USA). Cell nuclei were then stained with 40, 6-diamidino-2phenylindole (DAPI; 1:5000, Life Technologies, USA). Fluorescence images were captured by Olympus FV3000 confocal system (Olympus Corporation, Japan).

Protein solubility assay, SDS-PAGE, and immunoblotting Forty-eight hours after transfection, transfected cells were lysed in the mild lysis buffer (CelLytic ${ }^{\text {TM }}$ M, Sigma, USA). Lysates were centrifuged for $15 \mathrm{~min}$ 
with 12,000 r.p.m. by $4^{\circ} \mathrm{C}$ to separate soluble and insoluble fractions. The insoluble fraction was washed three times in the mild lysis buffer and resuspended with ultrasound homogenization. For western blots, $20 \mu \mathrm{g}$ of protein samples from HEK-293T cells or fibroblasts were separated by $10 \%$ SDS-polyacrylamide gel electrophoresis (SDS-PAGE). Specific bands were detected with anti-Myc (1:1000, ab18185, Abcam, China), anti- $\beta$-tubulin (1:5000, AC010, Abclonal, China), and anti-SORD (1:1000, ab189248, Abcam, China), respectively.

\section{Statistical analyses}

All the variables were presented as mean \pm s.d. The data were compared with a two-tailed Student's $t$ test or a one-way ANOVA using GraphPad Prism (GraphPad Software Inc.). $P$ values $<0.05$ were considered significant.

\section{Reporting summary}

Further information on research design is available in the Nature Research Reporting Summary linked to this article.

\section{DATA AVAILABILITY}

The data that support the findings of this study are available from the corresponding author upon reasonable request. Whole-exome sequencing data for the patient are deposited in the Sequence Read Archive (SRA) under accession code number PRJNA672732.

Received: 23 June 2020; Accepted: 25 November 2020; Published online: 04 January 2021

\section{REFERENCES}

1. Rossor, A. M., Kalmar, B., Greensmith, L. \& Reilly, M. M. The distal hereditary motor neuropathies. J. Neurol. Neurosurg. Psychiatry 83, 6-14 (2012).

2. Murphy, S. M. et al. Charcot-Marie-Tooth disease: frequency of genetic subtypes and guidelines for genetic testing. J. Neurol. Neurosurg. Psychiatry 83, 706-710 (2012).

3. Bansagi, B. et al. Genetic heterogeneity of motor neuropathies. Neurology $\mathbf{8 8}$ 1226-1234 (2017).

4. Dierick, I. et al. Relative contribution of mutations in genes for autosomal dominant distal hereditary motor neuropathies: a genotype-phenotype correlation study. Brain 131, 1217-1227 (2008).

5. Rossor, A. M., Polke, J. M., Houlden, H. \& Reilly, M. M. Clinical implications of genetic advances in Charcot-Marie-Tooth disease. Nat. Rev. Neurol. 9, 562-571 (2013).

6. Cortese, A. et al. Biallelic mutations in SORD cause a common and potentially treatable hereditary neuropathy with implications for diabetes. Nat. Genet. 52, 473-481 (2020)

7. Hellgren, M., Kaiser, C., de Haij, S., Norberg, A. \& Höög, J. O. A hydrogenbonding network in mammalian sorbitol dehydrogenase stabilizes the tetrameric state and is essential for the catalytic power. Cell Mol. Life Sci. 64 3129-3138 (2007).

8. Safi, S. Z., Qvist, R., Kumar, S., Batumalaie, K. \& Ismail, I. S. Molecular mechanisms of diabetic retinopathy, general preventive strategies, and novel therapeutic targets. Biomed. Res. Int. 2014, 801269 (2014).

9. $\mathrm{Ng}, \mathrm{T}$. F. et al. Effects of sorbitol dehydrogenase deficiency on nerve conduction in experimental diabetic mice. Diabetes 47, 961-966 (1998)

10. Li, J. Q., Dong, H. L., Chen, C. X. \& Wu, Z. Y. A novel WARS mutation causes distal hereditary motor neuropathy in a Chinese family. Brain 142, e49 (2019).

11. Li, L. X., Liu, G. L., Liu, Z. J., Lu, C. \& Wu, Z. Y. Identification and functional characterization of two missense mutations in NDRG1 associated with CharcotMarie-Tooth disease type 4D. Hum. Mutat. 38, 1569-1578 (2017).

12. Dong, H. L. et al. Genetic spectrum of MCM3AP and its relationship with phenotype of Charcot-Marie-Tooth disease. J. Peripher Nerv. Syst. 25, 107-111 (2020).

13. Chen, C. X. \& Dong, H. L. Genetic spectrum and clinical profiles in a southeast Chinese cohort of Charcot-Marie-Tooth disease. Clin. Genet. 96, 439-448 (2019).

14. Li, L. X., Dong, H. L., Xiao, B. G. \& Wu, Z. Y. A novel missense mutation in peripheral myelin protein-22 causes Charcot-Marie-Tooth Disease. Chin. Med. J. 130, 1779-1784 (2017).
15. Genomes Project, C. et al. A global reference for human genetic variation. Nature 526, 68-74 (2015).

16. Lek, M. et al. Analysis of protein-coding genetic variation in 60,706 humans Nature 536, 285-291 (2016).

17. Karczewski, K. J. \& Francioli, L. C. The mutational constraint spectrum quantified from variation in 141,456 humans. Nature 581, 434-443 (2020).

18. Ng, P. C. \& Henikoff, S. SIFT: Predicting amino acid changes that affect protein function. Nucleic Acids Res. 31, 3812-3814 (2003).

19. Adzhubei, I., Jordan, D. M. \& Sunyaev, S. R. Predicting functional effect of human missense mutations using PolyPhen-2. Curr. Protoc. Hum. Genet. Chapter 7 Unit7.20 (2013).

20. Schwarz, J. M., Cooper, D. N., Schuelke, M. \& Seelow, D. MutationTaster2: mutation prediction for the deep-sequencing age. Nat. Methods 11, 361-362 (2014).

21. Kircher, M. et al. A general framework for estimating the relative pathogenicity of human genetic variants. Nat. Genet. 46, 310-315 (2014).

22. Jian, X., Boerwinkle, E. \& Liu, X. In silico prediction of splice-altering single nucleotide variants in the human genome. Nucleic Acids Res. 42, 13534-13544 (2014).

\section{ACKNOWLEDGEMENTS}

This study was supported by Fundamental Research Funds for the Central Universities, Grant/Award Number: 2019XZZX001-01-04; the research foundation for distinguished scholar of Zhejiang University, Grant/Award Number: 188020-1938 10101/089. We would like to thank the patients and their family members for participating in this study.

\section{AUTHOR CONTRIBUTIONS}

Z.-Y.W. designed and conceived the study. H.-L.D. and J.-Q.L. performed the analysis of mutations in all the families. H.-L.D. performed the functional experiments and analysis of data. G.-L.L., H.Y., and Z.-Y.W. evaluated and diagnosed the patients. H.-L.D. and J.-Q.L. wrote the manuscript. Z.-Y.W. critically revised the manuscript. All authors participated in discussion of the final manuscript and interpretation of results.

\section{COMPETING INTERESTS}

The authors declare no competing interests.

\section{ADDITIONAL INFORMATION}

Supplementary information is available for this paper at https://doi.org/10.1038/ s41525-020-00165-6.

Correspondence and requests for materials should be addressed to Z.-Y.W

Reprints and permission information is available at http://www.nature.com/ reprints

Publisher's note Springer Nature remains neutral with regard to jurisdictional claims in published maps and institutional affiliations.

\footnotetext{
Open Access This article is licensed under a Creative Commons Attribution 4.0 International License, which permits use, sharing, adaptation, distribution and reproduction in any medium or format, as long as you give appropriate credit to the original author(s) and the source, provide a link to the Creative Commons license, and indicate if changes were made. The images or other third party material in this article are included in the article's Creative Commons license, unless indicated otherwise in a credit line to the material. If material is not included in the article's Creative Commons license and your intended use is not permitted by statutory regulation or exceeds the permitted use, you will need to obtain permission directly from the copyright holder. To view a copy of this license, visit http://creativecommons. org/licenses/by/4.0/.
}

(c) The Author(s) 2021 